\title{
On a Certain Method of Rejecting Games With Too Much Risk and Not Enough Expected Gain
}

\author{
Ewa Drabik \\ Warsaw University of Technology, Warsaw, Poland
}

\begin{abstract}
Many important economic decisions involve an element of risk. Risk aversion is a concept in economics, game theory, finance, and psychology related to the behavior of consumers, players, and investors under uncertainty. Loss aversion is an important component of a phenomenon that has been widely discussed in recent years. It refers to a tendency to feel the pain of a loss more acutely than the pleasure of an equal-sized gain. Many scientists have analyzed the problem of profitability in games. Some authors presented certain features which characterize "safe" games played once. Kahneman and Tversky (1991) showed that the ratio of loss aversion to gain attraction should amount to $1: 2$. The aim of this paper is to show an asymptotically efficient strategy which enables the risk-averse player to establish boundary variables of loss and gain at each stage of a repeated game.

Keywords: asymptotically efficient adaptive allocation rule, one-armed bandit problem, risk aversion, repeated games
\end{abstract}

\section{Introduction}

In some games the combined value of risk, losses, and threats exceeds any and all benefits the player could derive from the game. In such cases, a rationally behaving player should not participate in the competition. However, in many social games the participation is virtually mandatory, and it is often necessary to cooperate with a group of individuals sharing common goals (e.g. in a workplace environment). The stakes of such a game can be resources, a formal structure, and the individual players' positions in that structure. If one does not participate in the game - consciously or not - they can drop out of this structure. This principle is similar for many other market structures (i.e. financial market) whose existence depends mainly on the behavior of the structure's participants.

It is usually assumed that a rational player has an aversion to risk. Risk aversion is a long known concept in economics, game theory, finance, and psychology. It refers to the behavior of consumers, players, and investors operating under conditions of uncertainty. The degree of risk aversion can be expressed mathematically; the most known measures of risk aversion were introduced by Pratt (1964) and Arrow (1965). One of the most important issues is the problem of participation in undertakings, investments, or games, including those played on the market, which are characterized by high risk. From the point of view of the player (e.g. a market participant) it is extremely important to determine the maximum loss and the minimum

Ewa Drabik, professor, Faculty of Management, Warsaw University of Technology, Warsaw, Poland.

Correspondence concerning this article should be addressed to Ewa Drabik, Faculty of Management, Warsaw University of Technology, Narbutta 85, Warsaw 02-524, Poland. 
profit which will make them decide to participate in the game. These problems were discussed by numerous authors (Palacios-Huerta \& Serrano, 2006; Rabin, 2000; Tversky \& Kahneman, 1991).

The problem of risk aversion was raised and then developed by Kahneman and Tversky (1991) as a part of their study of prospect theory. Their theory states, among other things, that the economy is composed of not only regularities but also people, who do not always behave rationally. They also showed that market participants perceive a loss differently than an equal (when it comes to value) profit. In other words, the pain of a loss can be felt more acutely than the joy of an equal profit. In 2000 Rabin showed that if a player is a risk averse expected utility maximizer and rejects a given gamble of equally likely gain $g$ and loss $l, g>1>0$, over a given range of wealth level, then they will reject correspondingly larger gambles of gain $G$ and loss $L$. Palacios-Huerta and Serrano (2006) showed the relationship between risk attitudes for low- and high-stakes gambles.

As far as the rational approach to a game is concerned, it is of major importance to establish $l$ (loss) as well as $g$ (gain) at each stage. Loss aversion was introduced by Kehneman and Tversky (1979) as part of the more general "prospect theory". In 1991 they, as well as others, estimated the loss aversion to gain attraction ratio to be about 2:1. Many authors such as Rabin and Thaler (2001), Segal and Spivak (1990), and Epstein (1992) have discussed the problem of participation in games of different stakes, and examined the participants' attitude to risk in different contexts and with different utility functions.

The problem of rejecting "bad" games was also discussed by Palacios-Huerta and Serrano (2006). They identified some common features of safe games, and at the same time formulated a very important principle which determines the profit and loss thresholds for the game to be considered "safe" - this will be presented in a later section. Aumann and Serrano (2007), basing on the Arrow-Pratt risk aversion measures, defined the so-called riskiness indices whose properties were expressed, among other methods, with axioms.

The aforementioned study considered only single-stage games. The aim of this work is to present a strategy to determine profit and loss thresholds in games played repeatedly. This will be accomplished using an asymptotically efficient strategy which was originally used by Lai and Robbins (1985) to solve the one-armed bandit problem.

This paper is divided into sections in the following way. The second part presents some of the theory related to different attitudes of market participants to risk, as well as the principle of determining profit and loss thresholds at which one should not participate in a game played once (Palacios-Huerta \& Serrano, 2006). The third part presents a strategy for calculating profit and loss thresholds at which one should reject a multi-stage game at a specific phase in order for the game to be profitable.

\section{Research Design}

\section{Research Subject}

The paper aims at discussing an asymptotically efficient allocation rule which enables the risk-aversive player to establish loss/gain thresholds at each stage of a multi-stage game. The specific objective is to present measures of risk aversion that make it possible to assess the situation in the game.

\section{Theory and Hypotheses}

The allocation rule based on the one-armed bandit problem solution is asymptotically efficient. Therefore it results in successful rejection of so-called bad games. 


\section{Basic Information About Risk Aversion, Neutrality, Risk-Loving Player}

\section{Elements of the Expected Utility Hypothesis}

Risk aversion is a concept in economics, game theory, finance, and psychology related to the behavior of consumers, players, and investors under uncertainty.

The most famous measures of risk-aversion were introduced by Pratt (1964) and Arrow (1965). For a player (or decision maker) with wealth level $w$ and twice differentiable utility function $u$,

Definition 1. The Pratt-Arrow coefficient of absolute risk aversion, at wealth $w$, is:

$$
r_{A}(w, u)=-\frac{u^{\prime \prime}(w)}{u^{\prime}(w)}
$$

where $u$ is a twice differentiable Bernoulli utility function.

The coefficient of relative risk aversion (RRA) is:

$$
r_{r}(w, u)=w \cdot r_{A}(w, u)
$$

Absolute risk aversion is a measure of the player's reaction to uncertainty associated with absolute changes in affluence. Relative risk aversion expresses the player's attitude to the "uncertainty" associated with the relative changes (percentage) in affluence. A player whose risk aversion decreases along with an increase in affluence will spend more and more money on a risky game. There may also be differences in the players' degree of risk aversion.

The expected utility can be expressed as:

$$
V=\sum_{x} p(x) u(x)
$$

where

$u: X \rightarrow R$ elementary utility function (Bernoulli utility function);

$p_{i}$ probability of an outcome occurring (representing a probability in simple lottery).

A simple example to illustrate the subsequently discussed concepts is simple lottery. In this case the probability $p_{i}$ represents the probability of event $i$, while $n$ - tuple $L=\left(p_{1}, \ldots, p_{n}\right), \quad p_{i} \geq 0$ defines the lottery, while $i=1, \ldots, n$ and $\sum_{i} p_{i}=1$. Lottery can also be expressed geometrically as a point in an $n$-dimensional simplex.

$$
\Delta=\left\{p \in[0,1]^{n}: p_{1}+\ldots+p_{n}=1\right\} .
$$

Let $F_{z}(x)=P\{z \leq x\}$ be a distribution function corresponding to the random variable $z$. The player makes a selection according to the following guideline:

$F_{z}$ is preferable to $F_{y}$, i.e. $F_{z} \succ F_{y}$ if and only if

$$
V\left(F_{z}\right) \geq V\left(F_{y}\right) \text { where } V\left(F_{z}\right)=\int u(x) d F_{z}(x) \text { or equivalently } V\left(F_{z}\right)=\sum_{x} p(x) u(x) ;
$$

(analogically defining $V\left(F_{y}\right)$ ).

Let us assume that the random variable $z$ has two possible values $z_{1}$ or $z_{2}$. Let $p$ denote the probability of 
event $z_{1}$, and (1-p) the probability of event $z_{2} . u$ will denote the elemental utility function, whose expected value can be determined as follows:

$$
E(u)=p u\left(z_{1}\right)+(1-p) u\left(z_{2}\right) .
$$

The following denotations will be used.

$X$ - set of outcomes (real line $R$ );

$Z$-random variable;

$M$ - set of random variables;

$C(Z)$ - the income from lottery;

$\Pi(Z)=E(Z)-C(Z)$-risk premium.

It is assumed that the player qualifies as:

- Risk-averse:

A player is "risk-averse" if $C(Z)<E(Z)$ or $\Pi(Z)>0$ for all $Z \in M$;

- Risk-neutral:

A player is "risk-neutral" if $C(Z)=E(Z)$ or $\Pi(Z)=0$ for all $Z \in M$;

- Risk-loving:

A player is "risk-loving" if $C(Z)>E(Z)$ or $\Pi(Z)<0$ for all $Z \in M$.

The following theorem is true.

Theorem 1. Let $u: R \rightarrow R$ be an elementary utility function representing preferences $\geq_{h}$ over $M$ which is monotonically increasing.

Then

(1) $u$ is concave if and only if $\geq_{h}$ displays risk-aversion;

(2) $u$ is convex if and only if $\geq_{h}$ displays risk-loving;

(3) $u$ is linear if and only if displays risk-neutral.

Each player with utility $u$ can show:

- Decreasing absolute risk aversion (DARA):

$u$ displays absolute risk aversion if $\Pi(w, u)>\Pi(w+a, u)$ for all $a>0$.

- Increasing absolute risk aversion (IARA):

$u$ displays increasing absolute risk aversion if $\Pi(w, u)<\Pi(w+a, u)$ for all $a>0$.

- Constant absolute risk aversion (CARA):

$u$ displays constant absolute risk aversion if $\Pi(w, u)<\Pi(w+a, u)$ for all $a>0$.

Absolute risk aversion is a measure of the player's reaction to the uncertainty associated with absolute changes in affluence. Relative risk aversion expresses the player's relation to the "uncertainty" associated with the relative (percentage) changes in the level of his wealth. A player with a decreasing risk aversion along with an increase in affluence will spend more and more money on a risky game. Players may also have a varied degree of risk aversion.

The player has an absolute risk aversion if $r_{A}(w, u)$ is a decreasing function for a given $u($.$) . The selection$ of $u($.$) is therefore a very important problem in the discussed theory. Table 1$ presents examples of utility functions and their classification in terms of changes in absolute and relative risk aversion.

It should be emphasized that the utility functions presented in Table 1 are characteristic of a risk-averse 
investor. Other authors, e.g. Rabin (2000) presented other utility functions, such as

$$
u(w)=\frac{w^{1-\gamma}}{1-\gamma} \quad \gamma \geq 0
$$

The $u($.) selection problem is widely discussed, because it is assumed that not every player avoids risk to an equal degree. Studies on various issues make use of other utility functions, i.e. with different analytical forms and different properties resulting from the signs of the first and second derivative. It was however found that as a general rule, the basic characteristics of this function, assuming that the players maximize their expected utility, are not very sensitive to the type of individual risk propensity (represented by absolute and relative risk aversion). It should be added that besides the aforementioned Arrow-Pratt measures, other methods of measuring risk aversion also exist (Mass-Colell, Whinston, \& Green, 1995).

Table 1

Example Utility Functions and Their Classification in Terms of Changes in Absolute and Relative Risk Aversion

\begin{tabular}{llll}
\hline Utility function & & Absolute risk aversion & Relative risk aversion \\
\hline$u(w)=\ln w$ & & Decrease & Constant \\
$u(w)=(w+C)^{-\gamma}$ & $c>0, \quad \gamma \in(0,1)$ & Decrease & Increase \\
$u(w)=-e^{-\gamma w}$ & $\gamma>0$ & Constant & Increase \\
$u(w)=e^{-2 w^{-\frac{1}{2}}}$ & Decrease & Decrease \\
\hline
\end{tabular}

Source: based on Mass-Colell, Whinston, and Green (1995).

\section{"Safety" Games}

As was mentioned in the introduction, in the course of studies on the behavior of players, investors, consumers, and other market participants the problem of participation in high risk ventures developed naturally. Players are not willing to participate in games which bring not enough profit or too much loss. In 1991, Kahneman and Tversky proposed the term "loss aversion" instead of "risk aversion". In 2006, Palacios-Huerta and Serrano (2006) proposed and proved a theorem allowing to calculate the threshold value of loss $l$ to gain $g$ at which one can consider a game to be safe and participate in it.

Theorem 2. Let $u$ satisfy non-increasing absolute risk aversion. Let $I$ be an interval in the positive real line. For every $w \in I$

$$
\frac{1}{2} u(w+g)+\frac{1}{2} u(w-l)<u(w)
$$

there exists $a^{*}>0$ such that the absolute risk aversion coefficient $r_{A}(w, u)$ is greater than $a^{*}$ for all $w \in I$. Moreover, the highest such $a^{*}$ is the solution to the equation

$$
f(a)=e^{a l}+e^{-a g}-2=0
$$

where $l$ is loss, $g$ is gain.

Player rejects a game losing $l$ or gaining $g$.

The importance of theorem 2 is impossible to overstate. It must be noted, however, that it applies only to games played a single time. The next chapter will introduce a method that allows to determine the thresholds $U$ 
and $\mu$ in multi-stage games (games played repeatedly).

\section{Asymptotically Efficient Adaptive Allocation Rules and One-armed or Multi-armed Bandit}

Let us assume that the players participating in a particular game are risk-averse. This implies that the players feel the pain of a loss more acutely than the satisfaction from a gain of equal value. Therefore it is important to set threshold values of gain and loss which would satisfy the participants of games played repeatedly. This problem will be solved using a modified version of the strategy presented in the work of Lai and Robbins (1985) which was originally designed to solve the issue one-armed bandit problem.

The one-armed bandit is a mechanical or electrical machine, most frequently equipped with three drums holding a variety of card faces, used for gambling purposes. This simple machine has been known since 1887, when Charles Fey devised an automatic mechanism to make the trade of offered products more attractive. Since the machines proved to be very successful, in the middle of the 20th century the mechanical constructions were substituted by computerized devices, which were used in casinos. They were used to entertain and divert the ladies whose husbands and fiancés devoted themselves to card games.

First automats worked on a fully random basis. Currently, due to the application of software, the randomness is only partial. The participation in such games and generation of shots attracted the attention of many scholars who predominantly aimed to elaborate on profit-maximizing strategies. These strategies became attributable to many disciplines, such as economics, biology, control theory (compare available literature: Lai \& Robbins, 1985; Anantharam, Varaiya, \& Warland, 1987).

The three-reel slot machines invented in San Francisco in 1896 by Charles Fey underwent substantial changes over the course of the 20th century. For example, while classical slots were purely mechanical or electro-mechanical, modern ones are electronic and controlled by microprocessors with random number generators. Slot machines are the only casino games where evaluating the house advantage requires information not generally available to the gambling public. The slot machine is essentially a cabinet housing 3 or more narrow cylindrical drums, which are marked with symbols. Vertically placed on a common axis, the reels are caused to revolve freely when a player activates the machine and pulls a lever affixed to the side of the cabinet. Awards, which are paid out automatically, are based on the horizontal alignment of symbols when the spinning reels come to the position of inertial rest (Ethier, 2010).

Unstated but implicit in the definition was the requirement that the players insert one or more coins into a slot (hence the name "slot machine") in order to activate the machine. Nowadays almost all slots machines are electronic and controlled by microprocessors. The microprocessor is programmed with a random number generator that operates continually. The moment the player pulls the handle or presses the button to activate the machine, the most recently generated random numbers almost instantly determine the outcome. Despite all this, the underlying mathematics of the modern slot machine is much different from that of a classical machine.

Slot machines have a lot applications, for instance in statistics. One of them is the asymptotically efficient adaptive allocation rule which was assigned by Lai and Robbins (1985). In the following years the rule was further developed by other scholars and was renamed as the multi-armed bandit problem.

One of the strategies - the asymptotically efficient adaptive allocation rule-which is used in the field of statistics may be described as follows.

Let $w_{i}(i=1,2 \ldots)$ denote statistical populations (wealth, treatments, manufacturing processes etc.) specified by a univariate (or another, for instance normal) density function $f(w, \theta i)$ with respective measure $v$, 
where $f(.,$.$) is known and \theta i$ are unknown parameters belonging to a set $\Theta$. Assume that

$$
\int_{-\infty}^{\infty}|w| f(w, \theta) d v(w)<\infty \text { for all } \theta \in \Theta
$$

We sample $w_{1}, w_{2} \ldots$. sequentially from the $\mathrm{k}$ populations in order to achieve the greatest possible expected value $S_{n}=w_{1}+\ldots, w_{2}$ as $n \rightarrow \infty$. The multi-armed bandit problem is based on an imagined slot machine with $k \geq 2$ arms. When an arm is pulled the player wins a random reward. For each $\operatorname{arm} j$ there is an unknown probability distribution of the reward. The player's choices are in some way determined by the rewards of previous trials, so as to maximize the long-run total expected reward.

The method enables the establishment of random variables $l$ and $g$ in a more precise manner. Therefore, it can be counted among the asymptotically efficient adaptive allocation rules, which were applied for the first time by Lai and Robins (1985) to the multi-armed bandit problem with $k \geq 2$ arms. The rule may be explained as follows: in an n-stage game $w_{1}, w_{2}, \ldots, w_{n}$, on the basis of payouts (historical data) whose distribution is parameterized by an unknown parameter $\theta$ belonging to a known parameter space, two auxiliary statistics $\mu_{n}$ and $U_{n}$ are determined. Statistic $\mu_{n}$ represents the random variable $l$ and generally represents the average payout in the game (mean). $U_{n}$ may be considered as the upper bound of the confidence interval for a mean (a lower bound). The properties as well as construction of the above statistics will be presented below. In the following section loss $l$ is represented by statistic $\mu_{n}$, while gain $g$ is represented by statistic $U_{n}$.

It is assumed that during a specified number of stages $\left(T_{n} \leq n\right)$ the player continues to participate in the game regardless of the payouts received.

$$
\mu(\theta)=\int_{-\infty}^{+\infty} w f(w ; \theta) d v(w)
$$

Then

$$
E S_{n}=\sum_{j=0}^{k} \sum_{i=1}^{n} E\left(w_{i} I_{\left\{\varphi_{i}=j\right\}} \mid \mathfrak{J}_{i-1}\right)=\sum_{j=0}^{k} \mu(\theta) T_{n}(j)
$$

where

$$
T_{n}(j)=\sum_{i=1}^{n} I_{\left\{\varphi_{i}=j\right\}} \text { is the number of times that } \varphi \text { samples to stage } n \text {. The problem of maximizing } E S_{n} \text { is }
$$

therefore equivalent to that of minimizing regret

$$
R_{n}(\theta)=n \mu^{*}-E S_{n}=\sum_{j: \mu\left(\theta_{j}\right)<\mu^{*}}\left(\mu^{*}-\mu\left(\theta_{j}\right)\right) E T_{n}(j)
$$

where

$$
\mu^{*}=\max \left\{\mu\left(\theta_{0}\right), \mu\left(\theta_{1}\right)\right\}=\mu\left(\theta^{*}\right) \text { for some } \theta^{*} \in\left\{\theta_{0}, \theta_{1}\right\}
$$

Let $I(\theta, \lambda)$ denote the Kullback-Leibler number

$$
I(\theta, \lambda)=\int_{-\infty}^{\infty} \log \frac{f(w ; \theta)}{f(w ; \lambda)} \cdot f(w ; \theta) d v(w)
$$


such that $0<I(\theta, \lambda)<\infty$ whenever $\mu(\lambda)>\mu(\theta)$.

Generally regret is

$$
R_{n}(\theta) \approx\left\{\sum_{j: \mu\left(\theta_{j}\right)<\mu^{*}}\left(\mu^{*}-\mu\left(\theta_{j}\right)\right) / I\left(\theta_{j}, \theta^{*}\right)\right\} \log n \text { for } n \rightarrow \infty .
$$

The rule $\varphi$.

Let $w_{1}, w_{2}, \ldots$ be a sequence of random variables whose distribution is parametrized by unknown parameter $\theta \in \Theta$.

Let $g_{n t}: \mathfrak{R}^{t} \rightarrow \mathfrak{R}(n=1,2, \ldots ; t=1, \ldots, n)$ be a Borel function such that for every $\theta \in \Theta$

(W $P_{\theta}\left\{r \leq g_{n t}\left(w_{1}, \ldots, w_{t}\right)\right.$ for all $\left.t \leq n\right\}=1-o\left(n^{-1}\right)$

$\left(\mathrm{W}_{2}\right) \lim _{\varepsilon \rightarrow 0}\left(\limsup _{n \rightarrow \infty} \sum_{t=1}^{n} P_{\theta}\left\{g_{n t}\left(w_{1}, \ldots, w_{t}\right) \geq \mu(\lambda)-\varepsilon\right\} / \log n\right) \leq 1 / I(\theta, \lambda)$

whenever $\mu(\lambda)>\mu(\theta)$.

$\left(\mathrm{W}_{3}\right) \mathrm{g}_{n t}$ is nondecreasing in $n \geq t$ for every fixed $t=1,2, \ldots$

Let $h_{t}\left(w_{1}, \ldots, w_{t}\right)$ be a Borel function, $h_{t}: \mathfrak{R}^{t} \rightarrow \mathfrak{R}$, such that for every $\theta \in \Theta$

$\left(\mathrm{W}_{4}\right) h_{t} \leq g_{n t}$ for all $\theta \in \Theta$.

$\left(\mathrm{W}_{5}\right) P_{\theta}\left\{\max _{t}\left|h_{t}\left(w_{1}, \ldots, w_{t}\right)-\mu(\theta)\right|>\varepsilon\right\}=o\left(n^{-1}\right)$ for every $\varepsilon>0$.

Condition $\mathrm{W}_{5}$ can be satisfied for the average: $h_{t}\left(w_{1}, \ldots, w_{t}\right)=\left(w_{1}+\ldots+w_{t}\right) / t$ if $E_{\theta} w_{i}^{2}<\infty \quad(i=1, \ldots, t)$

and

$$
g_{n t}\left(w_{1}, \ldots, w_{t}\right)=\bar{w}_{t}+\sigma\left(2 a_{n t}\right)^{1 / 2} \text { for } n \geq t
$$

where $\sigma$ is standard deviation;

$a_{n t}(n=1,2, \ldots ; t=1, \ldots, n)$ is a positive constant such that for all $t, a_{n t}$ is nondecreasing for all $n \geq t$ and there exists $\varepsilon \rightarrow 0$ such that

$$
\left|a_{n t}-\frac{\log n}{t}\right| \leq \frac{\varepsilon(\log n)^{1 / 2}}{t^{1 / 2}} \text { for all } t \leq n
$$

Remark. If $f(.,$.$) denotes the density function of the normal distribution i.e.:$

$$
f(w ; \theta)=\left(2 \pi \sigma^{2}\right)^{-1 / 2} \exp \left\{-\frac{(w-\theta)^{2}}{2 \sigma^{2}}\right\}
$$

then

$$
I(\theta, \lambda)=\frac{(\theta-\lambda)^{2}}{2 \sigma^{2}}
$$

Let $w_{1}, \ldots, w_{T_{n}(j)}$ be the successive rewards obtained from $\operatorname{arm} j$ up to stage $n$. The upper confidence 
bound $U_{n}(j)$ and the point estimate $\mu_{\mathrm{n}}(j)$, the mean reward under $\operatorname{arm} j$, are given by

$$
\begin{gathered}
\mu_{n}(j)=h_{T_{n}(j)}\left(w_{1}, \ldots, w_{T_{n}(j)}\right) \\
U_{n}(j)=g_{n, T_{n}(j)}\left(w_{1}, \ldots, w_{T_{n}(j)}\right)
\end{gathered}
$$

Define the "leader" at stage $n$ as the population with the largest estimated mean among all population. The following rule $\varphi$ is applied to decide which arms to play.

In the first stage we observe $w_{1}, w_{2}, \ldots, w_{n}$ and compute $U_{n}(j)$ and $\mu_{\mathrm{n}}(j)$. Next, the player can take one of the following decisions.

In stage $(n+1)$ they make one of the following decisions:

(1) provided that in stage $n$ the player has not participated in the game and $\mu_{n} \leq 0$ they do not enter the game in stage $(n+1)$;

(2) provided that in stage $n$ the player has not participated in the game and $U_{n}<\mu_{n}$, they do not enter the game in stage $(n+1)$;

(3) provided that in stage $n$ the player has not participated in the game and $U_{n} \geq \mu_{n}$, they enter the game in stage $(n+1)$;

This is to prove that this type of strategy is asymptotically efficient in the sense of maximizing joint payouts (or minimizing the regret) in the game. What also seems interesting is the manner in which the above strategy influences risk-averse indices, which was presented in chapter 2.

Theorem. The rule $\varphi$ is asymptotically efficient.

Strategy $\varphi$ is asymptotically efficient, which means that the cost of such a game at $n \rightarrow \infty$ converges to an asymptote. This is particularly important for games where participation is mandatory and one can only opt out of participation in individual phases of the game.

The strategy presented in this work is intended for repeated games other than the one-armed bandit or stock market.

\section{Conclusions}

Some affluent people are seemingly fascinated by extreme risk and very often engage in activities which are not entirely legal, counting on a large profit. This raises a question related to psychology: why do they risk so much to have even more? There is no rational explanation. Keynes has stated that "when the development of a country becomes a side effect of casino operations, one should not expect positive results." Initially it was thought that financial markets are able to correct prices even if they are rising irrationally. However, an opinion of increasing popularity is that investors behave irrationally in moments of panic, often conducting speculations which are destructive, particularly to their competitors. While it is true that some mathematical models were developed on the assumption that investors rationally calculate risk and gain (e.g. Capital Asset Pricing Model-CAPM), predicting how to construct a wallet and set the price of financial instruments, they proved to be purely theoretical. The assumption of an efficient market caused the emergence of numerous "bubbles", and thus economics as a science was faced with some trouble. Financial markets are far from perfect. However, it is worth trying to counteract such peculiarities, relying among others on the help of mathematicians. The strategy

\footnotetext{
${ }^{1}$ https://en.wikiquote.org/wiki/John_Maynard_Keynes.
} 
presented in this work can contribute to this goal.

Games that initially bring high profits but are burdened with high risk are also dangerous from the point of view of potential investors (e.g. financial pyramids). Therefore, in the long term perspective, taking too much risk is inadvisable even if the game initially brings profits. It is better to withdraw from the game at a calculated stage, as discussed in the presented strategy $\phi$. If the game consistently brings losses, the decision seems obvious - it is best not to play.

\section{References}

Anantharam, V., Varaya, P., \& Warland, J. (1987). Asymptotically efficient allocation rules for multiarmed bandit problem with multiple plays. IEEE Transactions on Automatic Control, 32(11), 968-982.

Arrow, K. (1965). Aspects of the theory of risk-learning. Helsinki: Yrjö Johnssonin Sätiö.

Aumann, R. J., \& Serrano, R. (2007, July). An economic index of riskiness. 3th Spain Italy Netherlands Meeting on Game Theory (SING 3), Amsterdam.

Drabik, E. (2010). Asymptotically efficient adaptive allocation rule as a method of rejecting low and excessively high risk. Statistical Review, 1(57), 53-65.

Epstein, L. G. (1992). Behavior under risk: Recent developments. In. J. L. Laffont (Ed.), Theory and applications in advances in economic theory (Vol. II, pp. 1-63). Cambridge University Press.

Ethier, S. N. (2010). The doctrine of chances: Probabilistic aspects of gambling. Berlin-Heidelberg: Springer Verlag.

Kimball, M. S. (1991). Standard risk aversion. NBER Technical Working Paper, 99.

Mass-Colell, A., Whinston, M. D., \& Green, J. R. (1995). Microeconomic theory. New York: Oxford University Press.

Lai, T. L., \& Robbins, H. (1985). Asymptotically efficient adaptive allocation rules. Advanced in Applied Mathematics, 6, 4-22.

Palacios-Huerta, I., \& Serrano, R. (2006). Rejecting small gambles under expected utility. Economics Letters, 91, $250-259$.

Pratt, J. W. (1964). Risk aversion in the small and in the large. Econometrica, 1(32), 122-136.

Rabin, M. (2000). Risk aversion and expected utility: A calibration theorem. Econometrica, 68, 1287-1292.

Rabin, M., \& Thaler, R. H. (2001). Anomalies risk aversion. Journal of Economic Properties, 15(1), $219-232$.

Segal, U., \& Spivak, A. (1990). First order versus second order risk aversion. Journal of Economic Theory, 51, 111-125.

Tversky, A., \& Kahneman, R. (1991). Loss aversion in riskless choice: A reference-dependent model. Quarterly Journal of Economics, 106, 204-217. 